\title{
Energy-Efficient Data Fusion Technique and Applications in Wireless Sensor Networks
}

\author{
Yun Liu, ${ }^{1}$ Qing-An Zeng, ${ }^{2}$ and Ying-Hong Wang ${ }^{3}$ \\ ${ }^{1}$ Department of Electronic and Information Engineering, Key Laboratory of Communication and Information Systems, \\ Beijing Municipal Commission of Education, Beijing Jiaotong University, Beijing 100044, China \\ ${ }^{2}$ Department of Computer Systems Technology, North Carolina A\&T State University, Greensboro, NC 27411, USA \\ ${ }^{3}$ Department of Computer Science \& Information Engineering, Tamkang University, New Taipei City 25137, Taiwan \\ Correspondence should be addressed to Yun Liu; liuyun@bjtu.edu.cn
}

Received 21 April 2015; Accepted 21 April 2015

Copyright (c) 2015 Yun Liu et al. This is an open access article distributed under the Creative Commons Attribution License, which permits unrestricted use, distribution, and reproduction in any medium, provided the original work is properly cited.

Wireless Sensor Networks (WSNs) consist of a large number of source limited wireless sensor nodes for the purpose of data collection, processing, and transmission. Due to the limitations of sensor nodes' capabilities, especially the strictly limited energy, in-network data processing, such as data fusion which can significantly improve the energy-efficiency of the networks, is very important. This special issue mainly focuses on the latest research in the area of energy-efficient data fusion techniques and applications in WSNs. For this special issue, based on the review results we have selected seven papers that address the major issues of energy-efficient data fusion in WSNs and they are summarized as follows.

The paper "Minimum Cost Data Aggregation for Wireless Sensor Networks Computing Functions of Sensed Data" proposes a novel algorithm based on the key insight that there is a tradeoff in reducing costs between the local aggregation cost and finding a low cost path to the sink. The proposed algorithm can always empirically find the best tradeoff point. The authors also argue that the algorithm is applicable to many other similar types of problems. By simulation they show that significant cost savings can be achieved by the proposed algorithm.

The paper "Secure Data Fusion in Wireless Multimedia Sensor Networks via Compressed Sensing" proposes a novel secure data fusion strategy based on compressed image sensing and watermarking; namely, the algorithm exploits the sparsity in the image encryption. The approach relies on $l_{1}-$ norm regularization, common in compressive sensing, to enhance the detection of sparsity over wireless multimedia sensor networks. The resulting algorithms endow sensor nodes with learning abilities and allow them to learn the sparse structure from the still image data and also utilize the watermarking approach to achieve authentication mechanism.

The paper "An Improved Ant Colony Routing Algorithm for WSNs" proposes the improved ant colony algorithm to balance the energy consumption of networks. The improved ant colony routing algorithm is inspired by the Dijkstra algorithm changing the wireless sensor network undirected graph to directed graph and also by energy equilibrium consumption ideas. Through simulation and comparison with basic ant colony algorithms, it is obvious that improved algorithm can effectively balance energy consumption and extend the lifetime of WSNs.

The paper "GTDM: A DTN Routing on Noncooperative Game Theory in a City Environment" proposes a fixed sink station routing algorithm based structure and a more proper routing algorithm named Game Theory Based Decision Making (GTDM). GTDM shows decision making process for neighborhood selection and packet delivering strategy which is based on the noncooperative game theory method and city environment characteristics. The performance of GTDM is evaluated using numerical simulations under Working Day Movement (WDM) model and the results suggested that GTDM outperforms other traditional DTNs routing approaches, such as Epidemic and Prophet algorithm.

The paper "Design and Implementation of Energy Efficiency in HVAC Systems Based on Robust PID Control for 
Industrial Applications" develops the proportional-integralderivative (PID) programming which can effectively handle the discrete, nonlinear, and highly constrained optimization problems. Energy efficiency process has been made by controlling of alternative current (AC) drivers for ventilation and exhaust fans, according to supplied air flow capacity and differential air pressure between supplied and exhaust air. Supervisory controller software is developed by using programmable controllers and human machine interface (HMI) units. The new designed HVAC control system would have a saving potential of about $40 \%$ as compared to the existing operational settings, without any extra cost.

The paper "A Cluster-Based Fuzzy Fusion Algorithm for Event Detection in Heterogeneous Wireless Sensor Networks" proposes a cluster-based data fusion algorithm for event detection. The authors use $k$-means algorithm to form the nodes into clusters, which can significantly reduce the energy consumption of intracluster communication. Distances between cluster heads and event and energy of clusters are fuzzified thus to use a fuzzy logic to select the clusters that will participate in data uploading and fusion. Fuzzy logic method is also used by cluster heads for local decision, and then the local decision results are sent to the base station. Decision-level fusion for final decision of event is performed by base station according to the uploaded local decisions and fusion support degree of clusters calculated by fuzzy logic method. The effectiveness of this algorithm is demonstrated by simulation results.

Yun Liu

Qing-An Zeng

Ying-Hong Wang 

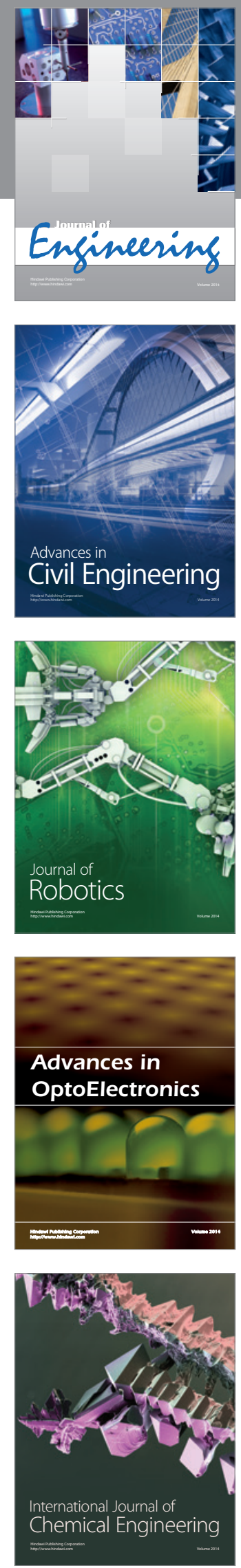

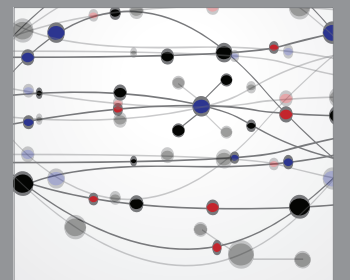

The Scientific World Journal
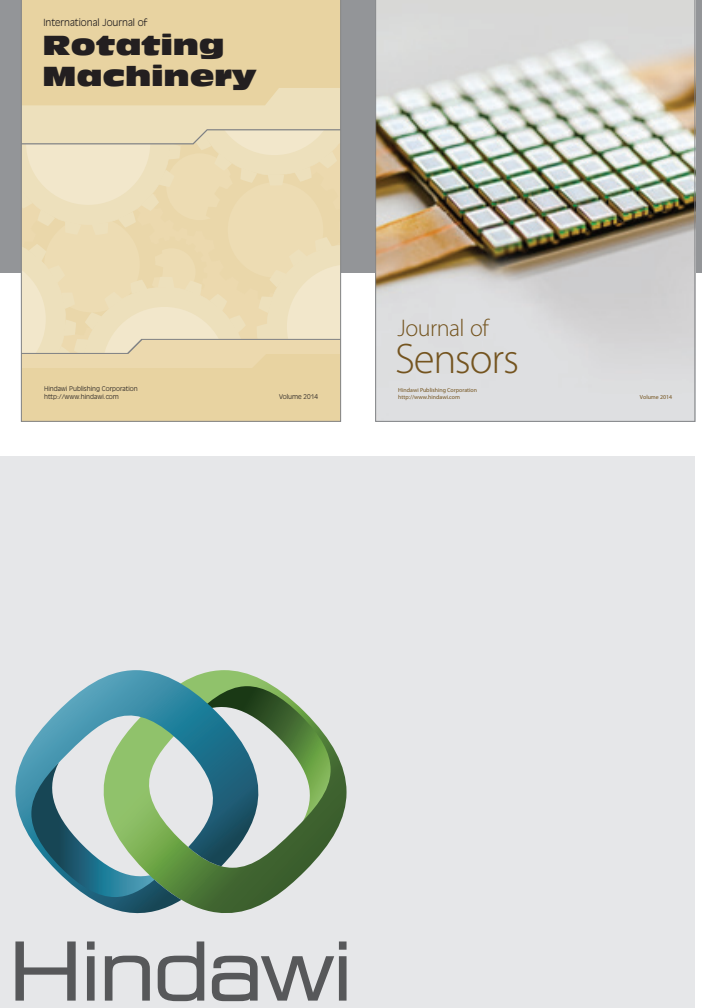

Submit your manuscripts at http://www.hindawi.com
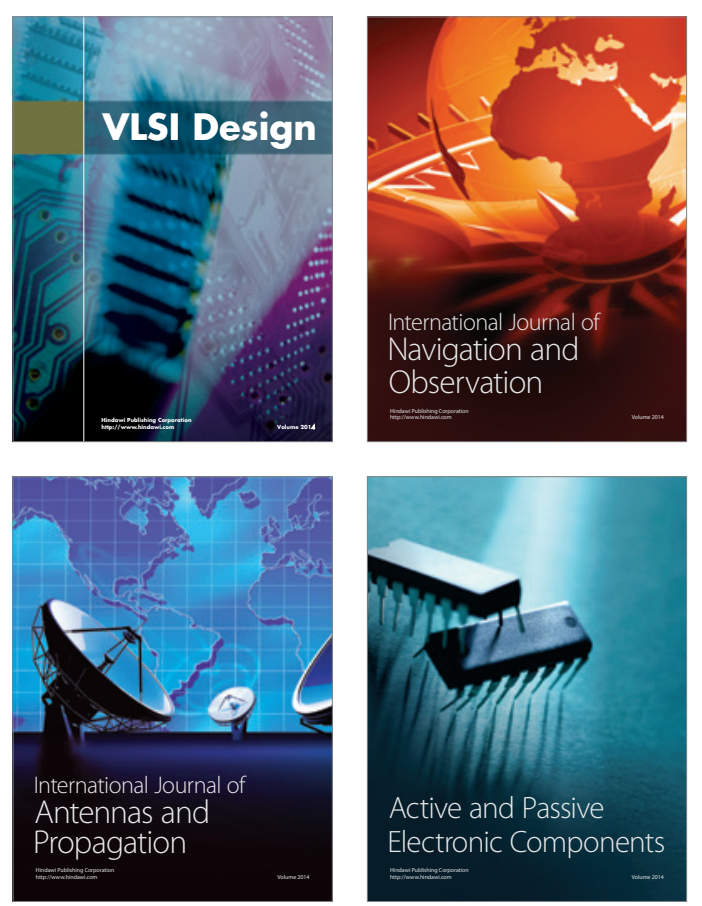
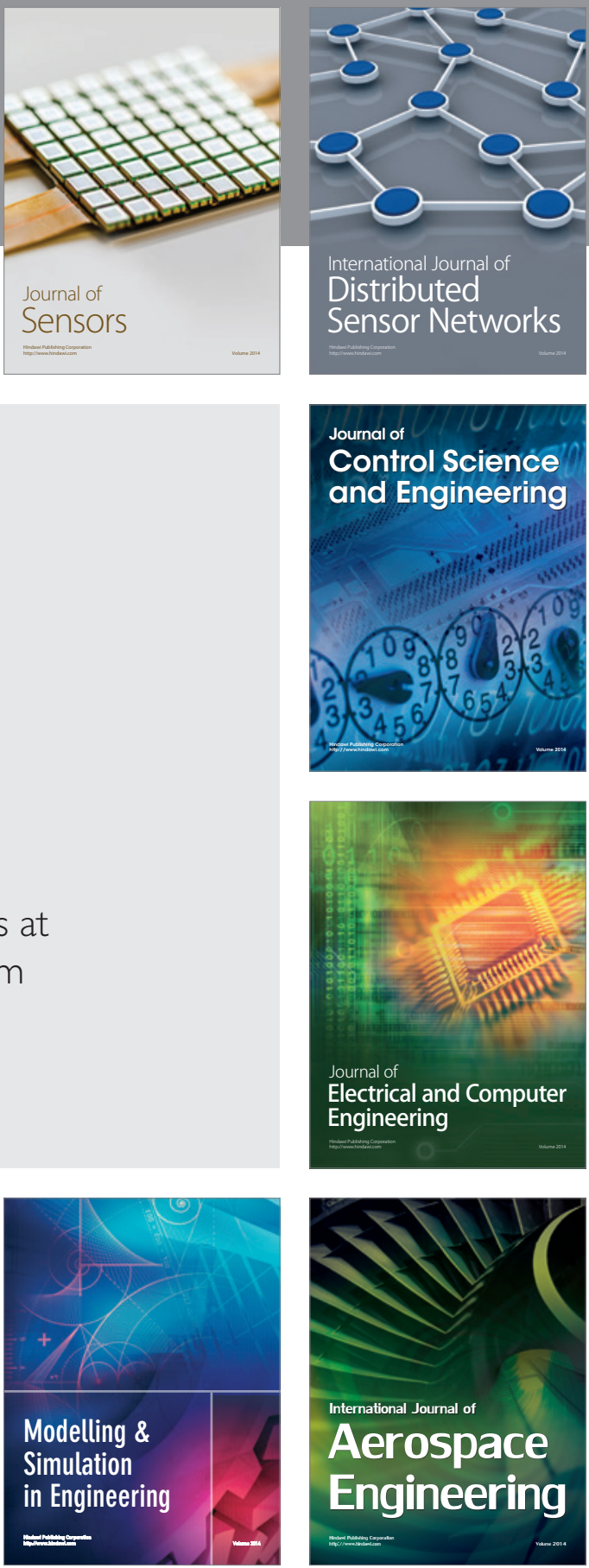

Journal of

Control Science

and Engineering
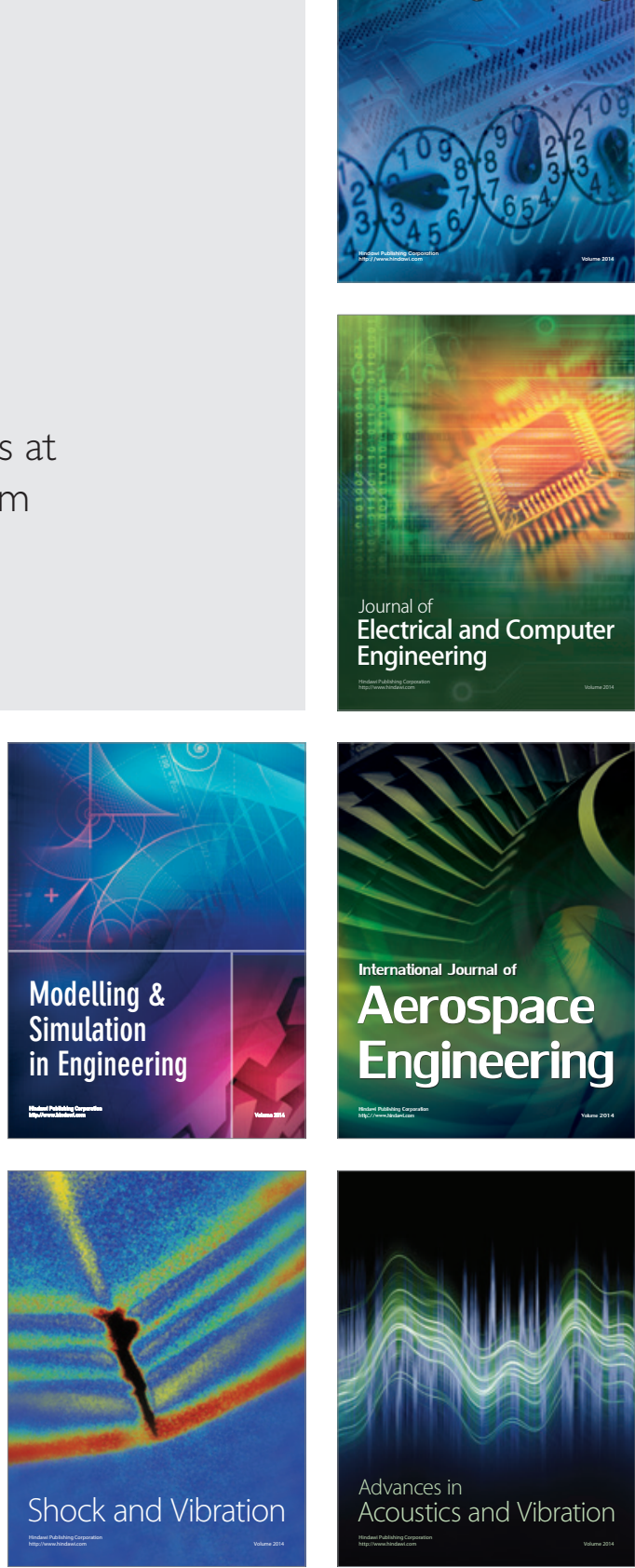